\title{
Mangrove Soil-Borne Trace Elements in Qi'ao Island: Implications for Understanding Terrestrial Input of Trace Elements into Part of the Pearl River Estuary
}

\author{
Anyi Niu ${ }^{1}$, Jiaojiao $\mathrm{Ma}^{1}$, Yifei Gao ${ }^{2}$, Songjun $\mathrm{Xu}^{1, *}$ and Chuxia Lin ${ }^{3, *}$ \\ 1 School of Geography, South China Normal University, Guangzhou 510631, China; \\ niuanyi@m.scnu.edu.cn (A.N.); jjma@m.scnu.edu.cn (J.M.) \\ 2 International Envirotech Limited, Hong Kong 999077, China; gaoyifei@internationalenvirotech.com \\ 3 Faculty of Science, Engineering and Built Environment, Deakin University, Burwood, VIC 3125, Australia \\ * Correspondence: xusj@scnu.edu.cn (S.X.); chu.lin@deakin.edu.au (C.L.); Tel.: +61-432-935-209 (C.L.)
}

Received: 31 January 2020; Accepted: 29 March 2020; Published: 3 April 2020

check for updates

\begin{abstract}
An investigation was conducted to characterize the trace element status of mangrove soils of Qi'ao Island in the Pearl River estuary. The results show that the spatial variation in the soil-borne trace elements in the investigated area was minor and most of the trace elements were at a level higher than those in other mangrove wetlands around the world, suggesting the mangrove soils of Qi'ao Island were heavily contaminated by trace elements transported from the Pearl River in the past two decades. $\mathrm{Zn}$ was closely related to $\mathrm{Pb}, \mathrm{Cu}, \mathrm{Cd}$, and $\mathrm{As}$, while some trace elements were not closely related to each other, indicating that they were derived from different sources. An integrated Nemerow pollution index of the surface soils at the 17 sampling locations ranged from 7.53 to 48.42 , values which all fall within the highest pollution category. Among the 17 sampling locations, six locations had an ecological risk index (ERI) greater than 300, and 12 locations had an Ecological Risk Index (ERI) greater than 600, indicating that most of the investigated locations were at high or very high ecological risk. The findings obtained from this study have implications for understanding the terrestrial inputs of trace elements into part of the Pearl River estuary. This understanding can be used to guide the development of management strategies for controlling the discharges of trace elements from the catchment area and managing the aquatic ecosystems in the Pearl River Estuary.
\end{abstract}

Keywords: mangrove; estuary; trace elements; soil; river; contamination

\section{Introduction}

Mangrove soils act as a sink for trace elements transported from inland areas by coastal rivers [1-4]. The elevated levels of trace elements in the mangrove soils could have adverse impacts on the mangrove growth, aquatic biota, and microbial activities in the mangrove habitats [5-8]. Understanding the level and spatial variation of trace elements in mangrove sediments/soils is essential for environmental risk assessment of the mangrove ecosystems. The relevant contamination information can also be used to indicate trace element loads of the river discharging into the estuary, thus helping environmental regulators and policy-makers to ensuring better environmental pollution control in the upstream catchment.

The Pearl River delta is one of the heavily industrialized areas in China, with petrochemical, electronic, electroplating, manufacturing, and textile industries being the major industrial sectors. These have resulted in discharge of substantial amounts of industrial wastewater into the Pearl River Estuary $[9,10]$.

The Pearl River is the third longest river in China, draining a total area of $453,690 \mathrm{~km}^{2}$. The large catchment size, coupled with a humid subtropical climate, makes the sediment load of the river great, 
resulting in the rapid growth of the Pearl River delta in the estuarine embayment. The sedimentation rate in the deltaic areas is generally very high and consequently disfavors colonization of mangrove plants. Therefore, mangroves are limited to islands or small bays along the coastline of the estuarine embayment that are far from the active distributaries. In the Pearl River estuary, trace element contamination in mangrove soils has been investigated in Mai Po and Futian in the Shenzhen Bay $[7,11,12]$. However, these locations are far from the active distributaries of the Pearl River and the trace elements in the contaminated mangrove soils are of local sources, such as discharges of municipal and industrial wastewater.

In this study, Qi'ao Island, which faces two major distributaries of the Pearl River, was selected to investigate the level and distribution of trace elements in the mangrove wetlands. The natural mangrove forest was largely cleared during the period from the early 1950s to the late 1990s. Since the establishment of the Qi'ao Natural Reserve in 1999, replanting of mangroves has resulted in an increase in the area of mangrove wetland from 32 ha to 600 ha. This has created favorable conditions for trapping suspended sediments discharged from the Pearl River. Therefore, the status of trace elements in the mangrove soils could, to a certain extent, reflect the characteristics of the riverine sediment-bearing trace elements. The aim of this study was to characterize the soil-borne trace elements in the mangrove soils and, based on the findings, evaluate their environmental risk and explore the links with the terrestrial input of the trace elements.

\section{Materials and Methods}

\subsection{Study Area}

Qi'ao Island $\left(113^{\circ} 36^{\prime} 41^{\prime \prime} \mathrm{E}-113^{\circ} 39^{\prime} 16^{\prime \prime} \mathrm{E} ; 22^{\circ} 23^{\prime} 41^{\prime \prime} \mathrm{N}-22^{\circ} 27^{\prime} 39^{\prime \prime} \mathrm{N}\right)$ has a total area of $23.8 \mathrm{~km}^{2}$. The area experiences a subtropical monsoon climate with annual average temperature and precipitation of $23.5^{\circ} \mathrm{C}$ and $1948.9 \mathrm{~mm}$, respectively. It has a mixed semidiurnal tidal cycle and the annual average salinity of the seawater is $1.82 \%$. Major types of rocks in the island include granite and granodiorite. The soils in the investigated area were classified as salic gleyic fluvisols according to the World Reference Base (WRB) soil classification system [13]. Major mangrove species include Sonneratia apetala, Sonneratia caseolaris (Linn.) Engl., Kandelia candel (Linn.) Druce, Heritiera littoralis Dryand., Parmentiera cerifera Seem., Bruguiera gymnorrhiza (Linn.) Savigny, Excoecaria agallocha Linn., Thespesia populnea (Linn.) Soland. ex Corr. and Acanthus ilicifolius Linn. Sp. [14-16].

\subsection{Soil Sampling Method}

Soil samples were collected from 17 selected locations with varying surface elevations (Figure 1). At each location, three replicate soil samples were collected from each of the three different soil layers $(0-10 \mathrm{~cm}, 10-20 \mathrm{~cm}$, and $20-30 \mathrm{~cm})$. A total of 153 soil samples was collected from the investigated area. Sampling Locations S1-S5 were within the upper intertidal zone; Sampling Locations S6-S13 were within the mid intertidal zone; and S14-S17 were within the lower intertidal zone. At each sampling location, 3 soil cores were taken to a depth of $30 \mathrm{~cm}$ using a soil corer. Soil samples were collected at $10 \mathrm{~cm}$ intervals (i.e., 3 samples from each soil profile) and put in sealable plastic bags. In the laboratory, the soil samples were air-dried, ground, and passed through a $0.149 \mathrm{~mm}$ sieve prior to analysis. 


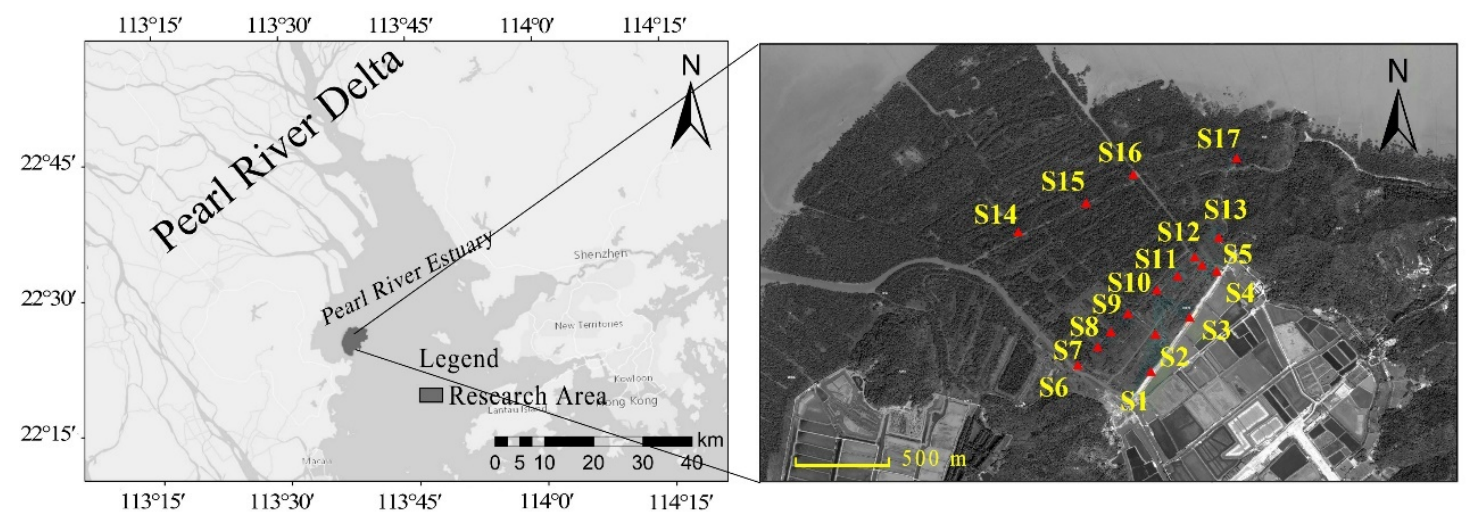

Figure 1. Maps showing the geographical location of the study area within the Pearl River estuary and the sampling locations within the study area

\subsection{Laboratory Methods}

The soil-borne trace elements were determined by inductively coupled plasma mass spectrometry (IPC-MS Agilent 7700) after digesting $0.13 \mathrm{~g}$ of soil with $8 \mathrm{~mL}$ of $\mathrm{HNO}_{3}$ and $2 \mathrm{~mL}$ of $\mathrm{HF}$ in a microwave digestion system $\left(\right.$ MARS $^{\mathrm{TM}}$ 6). The soil analysis was performed by the accredited laboratory of the Nanjing Soil Research Institute Analytical Service, Chinese Academy of Sciences.

\subsection{Evaluation of Soil Environmental Quality}

The environmental quality of the mangrove soils was assessed by a modified integrated Nemerow pollution index. The Nemerow pollution index (NPI) was calculated using the following formula [17]:

$$
\mathrm{NPI}=\mathrm{Ci} / \mathrm{Bi}
$$

where $C_{i}$ is the observed concentration of a trace element in the investigated soil, while $B_{i}$ represents the regional background value of that trace element.

By taking into account all the trace elements contained in the soil, the integrated Nemerow pollution index (INPI) can be obtained as follows [18]:

$$
\mathrm{INPI}=\left[\left(N P I_{\text {ave }}\right)^{2}+\frac{\left(N P I_{\max }\right)^{2}}{2}\right]^{0.5}
$$

where $N P I_{\text {ave }}$ denotes the mean value of the Nemerow pollution index for all the trace elements of concern while $N P I_{\max }$ represents the maximum value of the Nemerow pollution index among these trace elements. Five pollution categories were set (Table 1) according to Hou et al. [18].

Table 1. Classification system for pollution level of the mangrove soils based on integrated Nemerow pollution index (INPI).

\begin{tabular}{ccc}
\hline Pollution Category & INPI & Degree of Pollution \\
\hline 1 & $<0.7$ & No pollution \\
2 & $0.7-1$ & Alert \\
3 & $1-2$ & Slight pollution \\
4 & $2-3$ & Medium pollution \\
5 & $>3$ & Heavy pollution \\
\hline
\end{tabular}

The soil environmental quality was also assessed against the ecological risk index (ERI) using the following formula $[19,20]$ :

$$
\mathrm{ERI}=\sum E_{r}^{i}
$$




$$
\sum E_{r}^{i}=\sum T_{i} \times C_{i} / B_{i}
$$

where $E_{r}^{i}$ represents an individual ecological risk factor, $C_{i}$ is the observed concentration of a trace element in the investigated soil, and $B_{i}$ represents the regional background value of that trace element. $T_{i}$ is the toxicity coefficient of a trace element. For $\mathrm{Hg}, \mathrm{Cd}, \mathrm{As}, \mathrm{Ni}, \mathrm{Cu}, \mathrm{Pb}, \mathrm{Cr}$ and $\mathrm{Zn}, T_{i}$ is set at 40, 30, $10,5,5,5,2$, and 1, respectively [21]. Four ERI categories were set for this study (Table 2) according to Guo et al. [20]

Table 2. Classification system for ecological risk of the mangrove soils based on ecological risk index.

\begin{tabular}{ccc}
\hline Category & ERI & Ecological Risk Level \\
\hline 1 & $<150$ & low \\
2 & $150-300$ & medium \\
3 & $300-600$ & high \\
4 & $>600$ & very high \\
\hline
\end{tabular}

\section{Results}

\subsection{Upper Intertidal Zone}

On average, the concentration of the investigated trace elements was in the following decreasing order: $\mathrm{Zn}>\mathrm{Cr}>\mathrm{Pb}>\mathrm{Cu}>\mathrm{As}>\mathrm{Ni}>\mathrm{Cd}>\mathrm{Hg}$. In general, both horizontal and vertical variations in all the investigated trace elements were not remarkable, although exceptional high or low values were observed at some locations or soil layers. For example, $\mathrm{Cd}$ was several times higher in the surface soil layer at Location S1 than in the bottom soil layer at the same location and the soil layers at other locations. Similarly, Hg was several times higher in the surface soil layer at Location S4 than in the bottom soil layer at the same location and the soil layers at other locations (Table 3).

Table 3. Mangrove soil-borne trace elements $(\mathrm{mg} / \mathrm{kg})$ in the upper intertidal zone.

\begin{tabular}{|c|c|c|c|c|c|c|c|c|c|}
\hline Location & Depth & $\mathrm{Cr}$ & $\mathbf{N i}$ & $\mathrm{Cu}$ & $\mathrm{Zn}$ & As & $\mathrm{Cd}$ & $\mathrm{Hg}$ & $\mathbf{P b}$ \\
\hline \multirow{3}{*}{ S1 } & $0-10 \mathrm{~cm}$ & $\begin{array}{c}115.59 \pm \\
17.82\end{array}$ & $\begin{array}{c}31.57 \pm \\
4.23\end{array}$ & $\begin{array}{c}63.62 \pm \\
5.37\end{array}$ & $\begin{array}{c}94.63 \pm \\
7.63\end{array}$ & $\begin{array}{c}56.78 \pm \\
33.11\end{array}$ & $\begin{array}{c}5.51 \pm \\
2.90\end{array}$ & $\begin{array}{c}0.53 \pm \\
0.03\end{array}$ & $\begin{array}{c}59.90 \pm \\
19.54\end{array}$ \\
\hline & $\begin{array}{c}10-20 \\
\mathrm{~cm}\end{array}$ & $\begin{array}{c}104.03 \pm \\
0.07\end{array}$ & $\begin{array}{c}28.97 \pm \\
1.66\end{array}$ & $\begin{array}{c}59.67 \pm \\
3.62\end{array}$ & $\begin{array}{c}107.18 \pm \\
33.22\end{array}$ & $\begin{array}{c}100.00 \pm \\
49.75\end{array}$ & $\begin{array}{c}3.83 \pm \\
1.89\end{array}$ & $\begin{array}{c}0.69 \pm \\
0.27\end{array}$ & $\begin{array}{c}58.51 \pm \\
15.08\end{array}$ \\
\hline & $\begin{array}{l}20-30 \\
\mathrm{~cm}\end{array}$ & $\begin{array}{c}92.52 \pm \\
4.81\end{array}$ & $\begin{array}{c}25.53 \pm \\
1.87\end{array}$ & $\begin{array}{c}39.67 \pm \\
3.05\end{array}$ & $\begin{array}{c}97.84 \pm \\
6.05\end{array}$ & $\begin{array}{c}20.37 \pm \\
2.38\end{array}$ & $\begin{array}{c}0.46 \pm \\
0.04\end{array}$ & $\begin{array}{c}0.41 \pm \\
0.12\end{array}$ & $\begin{array}{c}31.90 \pm \\
4.50\end{array}$ \\
\hline \multirow{3}{*}{ S2 } & $0-10 \mathrm{~cm}$ & $\begin{array}{c}89.19 \pm \\
2.88\end{array}$ & $\begin{array}{c}26.50 \pm \\
0.31\end{array}$ & $\begin{array}{c}35.82 \pm \\
1.99\end{array}$ & $\begin{array}{c}122.37 \pm \\
13.55\end{array}$ & $\begin{array}{c}20.92 \pm \\
0.67\end{array}$ & $\begin{array}{c}0.49 \pm \\
0.02\end{array}$ & $\begin{array}{c}0.16 \pm \\
0.01\end{array}$ & $\begin{array}{c}36.32 \pm \\
0.40\end{array}$ \\
\hline & $\begin{array}{c}10-20 \\
\mathrm{~cm}\end{array}$ & $\begin{array}{c}90.08 \pm \\
2.48\end{array}$ & $\begin{array}{c}26.09 \pm \\
0.77\end{array}$ & $\begin{array}{c}35.44 \pm \\
1.99\end{array}$ & $\begin{array}{c}102.21 \pm \\
4.76\end{array}$ & $\begin{array}{c}21.12 \pm \\
0.38\end{array}$ & $\begin{array}{c}0.51 \pm \\
0.01\end{array}$ & $\begin{array}{c}0.56 \pm \\
0.45\end{array}$ & $\begin{array}{c}36.69 \pm \\
1.40\end{array}$ \\
\hline & $\begin{array}{c}20-30 \\
\mathrm{~cm}\end{array}$ & $\begin{array}{c}94.39 \pm \\
2.02\end{array}$ & $\begin{array}{c}26.88 \pm \\
0.42\end{array}$ & $\begin{array}{c}41.62 \pm \\
3.00\end{array}$ & $\begin{array}{c}107.18 \pm \\
0.80\end{array}$ & $\begin{array}{c}23.25 \pm \\
1.22\end{array}$ & $\begin{array}{c}0.59 \pm \\
0.05\end{array}$ & $\begin{array}{c}0.37 \pm \\
0.22\end{array}$ & $\begin{array}{c}40.21 \pm \\
1.17\end{array}$ \\
\hline \multirow{3}{*}{ S3 } & $0-10 \mathrm{~cm}$ & $\begin{array}{c}80.93 \pm \\
1.69\end{array}$ & $\begin{array}{c}22.24 \pm \\
0.00\end{array}$ & $\begin{array}{c}35.78 \pm \\
1.77\end{array}$ & $\begin{array}{c}93.23 \pm \\
11.35\end{array}$ & $\begin{array}{c}20.90 \pm \\
0.76\end{array}$ & $\begin{array}{c}0.41 \pm \\
0.01\end{array}$ & $\begin{array}{c}0.22 \pm \\
0.00\end{array}$ & $\begin{array}{c}31.97 \pm \\
0.55\end{array}$ \\
\hline & $\begin{array}{c}10-20 \\
\mathrm{~cm}\end{array}$ & $\begin{array}{c}86.15 \pm \\
3.41\end{array}$ & $\begin{array}{c}29.00 \pm \\
1.72\end{array}$ & $\begin{array}{c}45.88 \pm \\
7.41\end{array}$ & $\begin{array}{c}122.37 \pm \\
2.62\end{array}$ & $\begin{array}{c}25.73 \pm \\
6.18\end{array}$ & $\begin{array}{c}0.74 \pm \\
0.14 \\
\end{array}$ & $\begin{array}{c}0.24 \pm \\
0.03\end{array}$ & $\begin{array}{c}38.20 \pm \\
5.06\end{array}$ \\
\hline & $\begin{array}{c}20-30 \\
\mathrm{~cm}\end{array}$ & $\begin{array}{c}93.79 \pm \\
2.39\end{array}$ & $\begin{array}{c}39.70 \pm \\
11.42\end{array}$ & $\begin{array}{c}47.96 \pm \\
4.63\end{array}$ & $\begin{array}{c}128.05 \pm \\
1.86\end{array}$ & $\begin{array}{c}23.28 \pm \\
0.20\end{array}$ & $\begin{array}{c}0.90 \pm \\
0.30\end{array}$ & $\begin{array}{c}0.20 \pm \\
0.04\end{array}$ & $\begin{array}{c}45.85 \pm \\
8.72\end{array}$ \\
\hline
\end{tabular}


Table 3. Cont.

\begin{tabular}{cccccccccc}
\hline Location & Depth & $\mathbf{C r}$ & $\mathbf{N i}$ & $\mathbf{C u}$ & $\mathbf{Z n}$ & $\mathbf{A s}$ & $\mathbf{C d}$ & $\mathbf{H g}$ & $\mathbf{P b}$ \\
\hline \multirow{4}{*}{ S4 } & $0-10 \mathrm{~cm}$ & $91.40 \pm$ & $28.22 \pm$ & $32.18 \pm$ & $100.41 \pm$ & $17.78 \pm$ & $0.61 \pm$ & $2.81 \pm$ & $35.89 \pm$ \\
& & 1.10 & 3.60 & 0.69 & 10.08 & 0.93 & 0.04 & 2.19 & 2.14 \\
\cline { 2 - 10 } & $10-20$ & $102.47 \pm$ & $25.42 \pm$ & $34.43 \pm$ & $110.72 \pm$ & $35.76 \pm$ & $1.55 \pm$ & $0.88 \pm$ & $43.69 \pm$ \\
& $\mathrm{cm}$ & 5.42 & 1.37 & 2.75 & 20.42 & 16.32 & 0.99 & 0.35 & 8.82 \\
\cline { 2 - 10 } & $20-30$ & $107.20 \pm$ & $29.43 \pm$ & $36.70 \pm$ & $129.14 \pm$ & $45.67 \pm$ & $1.72 \pm$ & $0.67 \pm$ & $53.03 \pm$ \\
& $\mathrm{cm}$ & 8.44 & 3.60 & 2.57 & 24.53 & 24.33 & 1.02 & 0.16 & 11.56 \\
\hline \multirow{5}{*}{ S5 } & $0-10 \mathrm{~cm}$ & $93.60 \pm$ & $23.82 \pm$ & $30.21 \pm$ & $85.84 \pm$ & $48.82 \pm$ & $0.80 \pm$ & $0.18 \pm$ & $49.83 \pm$ \\
& & 4.61 & 1.07 & 0.70 & 2.54 & 26.90 & 0.18 & 0.03 & 3.71 \\
\cline { 2 - 10 } & $10-20$ & $112.71 \pm$ & $30.63 \pm$ & $38.09 \pm$ & $106.42 \pm$ & $22.79 \pm$ & $0.77 \pm$ & $0.23 \pm$ & $45.53 \pm$ \\
& $\mathrm{cm}$ & 0.93 & 1.41 & 1.82 & 4.06 & 1.62 & 0.05 & 0.03 & 4.23 \\
\cline { 2 - 10 } & $20-30$ & $103.66 \pm$ & $26.31 \pm$ & $34.09 \pm$ & $111.96 \pm$ & $21.49 \pm$ & $0.68 \pm$ & $0.24 \pm$ & $40.82 \pm$ \\
& $\mathrm{cm}$ & 3.13 & 0.38 & 1.27 & 6.55 & 0.09 & 0.05 & 0.03 & 1.59 \\
\hline \multirow{2}{*}{ Average } & & $97.37 \pm$ & $28.02 \pm$ & $40.81 \pm$ & $108.60 \pm$ & $32.90 \pm$ & $1.30 \pm$ & $0.57 \pm$ & $43.34 \pm$ \\
& & 1.90 & 0.95 & 1.58 & 3.56 & 5.01 & 0.30 & 0.17 & 2.15 \\
\hline
\end{tabular}

\subsection{Mid Intertidal Zone}

In general, marked horizontal and vertical variations in the investigated trace elements were not observed. However, the subsoil layer $(10-20 \mathrm{~cm})$ tended to have a higher concentration of $\mathrm{Zn}$ and As, as compared to the surface soil layer $(0-10 \mathrm{~cm})$ and the bottom soil layer $(20-30 \mathrm{~cm})$ at most of the sampling locations. An unusually high concentration of $\mathrm{Cd}$ was recorded at Location S8 and an unusually high concentration of $\mathrm{Hg}$ was detected at Location S11. The mean values of various trace elements were comparable to those in the upper intertidal zone with a similar decreasing order being observed: $\mathrm{Zn}>\mathrm{Cr}>\mathrm{Pb}>\mathrm{Cu}>\mathrm{As}>\mathrm{Ni}>\mathrm{Cd}>\mathrm{Hg}$ (Table 4).

Table 4. Mangrove soil-borne trace elements $(\mathrm{mg} / \mathrm{kg})$ in the mid intertidal zone.

\begin{tabular}{cccccccccc}
\hline Sample & Depth & $\mathbf{C r}$ & $\mathbf{N i}$ & $\mathbf{C u}$ & $\mathbf{Z n}$ & $\mathbf{A s}$ & $\mathbf{C d}$ & $\mathbf{H g}$ & $\mathbf{P b}$ \\
\hline \multirow{5}{*}{ S6 } & $0-10 \mathrm{~cm}$ & $95.45 \pm$ & $31.66 \pm$ & $41.39 \pm$ & $90.20 \pm$ & $26.06 \pm$ & $0.62 \pm$ & $0.19 \pm$ & $39.62 \pm$ \\
& & 2.52 & 0.32 & 7.39 & 1.60 & 0.52 & 0.16 & 0.04 & 5.41 \\
\cline { 2 - 10 } & $10-20$ & $100.84 \pm$ & $29.00 \pm$ & $37.78 \pm$ & $106.08 \pm$ & $32.15 \pm$ & $0.60 \pm$ & $0.18 \pm$ & $40.38 \pm$ \\
& $\mathrm{cm}$ & 1.57 & 0.88 & 1.93 & 4.56 & 9.64 & 0.04 & 0.02 & 1.83 \\
\cline { 2 - 10 } & $20-30$ & $92.82 \pm$ & $29.02 \pm$ & $34.91 \pm$ & $82.33 \pm$ & $21.85 \pm$ & $2.32 \pm$ & $0.14 \pm$ & $41.27 \pm$ \\
& $\mathrm{cm}$ & 2.31 & 1.49 & 0.91 & 3.44 & 0.58 & 1.89 & 0.01 & 10.87 \\
\hline \multirow{5}{*}{ S7 } & $0-10 \mathrm{~cm}$ & $93.31 \pm$ & $27.53 \pm$ & $33.48 \pm$ & $103.08 \pm$ & $20.40 \pm$ & $0.49 \pm$ & $0.15 \pm$ & $37.84 \pm$ \\
& 1.79 & 0.05 & 0.99 & 2.81 & 0.96 & 0.01 & 0.01 & 0.38 \\
\cline { 2 - 10 } & $10-20$ & $101.17 \pm$ & $30.61 \pm$ & $46.08 \pm$ & $181.25 \pm$ & $106.00 \pm$ & $2.26 \pm$ & $0.16 \pm$ & $81.20 \pm$ \\
& $\mathrm{cm}$ & 4.47 & 0.43 & 6.83 & 72.36 & 83.20 & 1.63 & 0.04 & 41.36 \\
\cline { 2 - 10 } & $20-30$ & $100.95 \pm$ & $28.31 \pm$ & $43.14 \pm$ & $92.49 \pm$ & $22.68 \pm$ & $0.48 \pm$ & $0.13 \pm$ & $35.67 \pm$ \\
& $\mathrm{cm}$ & 1.15 & 0.53 & 6.83 & 4.69 & 0.85 & 0.02 & 0.03 & 0.62 \\
\hline \multirow{5}{*}{ S8 } & $0-10 \mathrm{~cm}$ & $90.07 \pm$ & $29.98 \pm$ & $44.21 \pm$ & $109.70 \pm$ & $30.36 \pm$ & $4.03 \pm$ & $0.39 \pm$ & $64.78 \pm$ \\
& & 2.68 & 1.50 & 7.37 & 9.83 & 8.56 & 3.46 & 0.06 & 24.19 \\
\cline { 2 - 10 } & $10-20$ & $94.22 \pm$ & $27.89 \pm$ & $43.36 \pm$ & $128.33 \pm$ & $51.37 \pm$ & $4.68 \pm$ & $0.33 \pm$ & $74.49 \pm$ \\
& $\mathrm{cm}$ & 4.34 & 1.68 & 5.46 & 7.07 & 27.64 & 3.38 & 0.03 & 29.23 \\
\cline { 2 - 10 } & $20-30$ & $95.21 \pm$ & $27.33 \pm$ & $37.14 \pm$ & $107.32 \pm$ & $24.66 \pm$ & $2.26 \pm$ & $0.25 \pm$ & $47.33 \pm$ \\
& $\mathrm{cm}$ & 1.74 & 0.35 & 1.69 & 8.56 & 3.04 & 1.61 & 0.06 & 12.18 \\
\hline
\end{tabular}


Table 4. Cont.

\begin{tabular}{|c|c|c|c|c|c|c|c|c|c|}
\hline Sample & Depth & $\mathrm{Cr}$ & $\mathrm{Ni}$ & $\mathrm{Cu}$ & $\mathrm{Zn}$ & As & $\mathrm{Cd}$ & $\mathrm{Hg}$ & $\mathrm{Pb}$ \\
\hline \multirow{3}{*}{ S9 } & $0-10 \mathrm{~cm}$ & $\begin{array}{c}97.92 \pm \\
1.89\end{array}$ & $\begin{array}{c}26.90 \pm \\
0.33\end{array}$ & $\begin{array}{c}34.77 \pm \\
0.47\end{array}$ & $\begin{array}{c}95.15 \pm \\
0.60\end{array}$ & $\begin{array}{c}21.47 \pm \\
0.16\end{array}$ & $\begin{array}{c}0.48 \pm \\
0.02\end{array}$ & $\begin{array}{c}0.33 \pm \\
0.08\end{array}$ & $\begin{array}{c}37.19 \pm \\
1.16\end{array}$ \\
\hline & $\begin{array}{c}10-20 \\
\mathrm{~cm}\end{array}$ & $\begin{array}{c}98.50 \pm \\
4.37\end{array}$ & $\begin{array}{c}29.08 \pm \\
0.78\end{array}$ & $\begin{array}{c}41.45 \pm \\
2.14\end{array}$ & $\begin{array}{c}177.89 \pm \\
32.77\end{array}$ & $\begin{array}{c}78.47 \pm \\
31.50\end{array}$ & $\begin{array}{c}2.58 \pm \\
1.06\end{array}$ & $\begin{array}{c}0.23 \pm \\
0.04\end{array}$ & $\begin{array}{c}62.33 \pm \\
12.11\end{array}$ \\
\hline & $\begin{array}{c}20-30 \\
\mathrm{~cm}\end{array}$ & $\begin{array}{c}98.95 \pm \\
5.53\end{array}$ & $\begin{array}{c}29.41 \pm \\
1.66\end{array}$ & $\begin{array}{c}38.98 \pm \\
2.98\end{array}$ & $\begin{array}{c}102.79 \pm \\
5.64\end{array}$ & $\begin{array}{c}22.19 \pm \\
0.85\end{array}$ & $\begin{array}{c}0.66 \pm \\
0.03\end{array}$ & $\begin{array}{c}0.22 \pm \\
0.04\end{array}$ & $\begin{array}{c}37.00 \pm \\
0.49\end{array}$ \\
\hline \multirow{3}{*}{$\mathrm{S} 10$} & $0-10 \mathrm{~cm}$ & $\begin{array}{c}90.08 \pm \\
0.76\end{array}$ & $\begin{array}{c}25.59 \pm \\
0.88\end{array}$ & $\begin{array}{c}37.30 \pm \\
2.07\end{array}$ & $\begin{array}{c}105.57 \pm \\
9.01\end{array}$ & $\begin{array}{c}21.03 \pm \\
1.42\end{array}$ & $\begin{array}{c}0.50 \pm \\
0.00\end{array}$ & $\begin{array}{c}0.37 \pm \\
0.09\end{array}$ & $\begin{array}{c}40.07 \pm \\
4.72\end{array}$ \\
\hline & $\begin{array}{c}10-20 \\
\mathrm{~cm}\end{array}$ & $\begin{array}{c}100.86 \pm \\
5.97\end{array}$ & $\begin{array}{c}26.89 \pm \\
1.13\end{array}$ & $\begin{array}{c}35.79 \pm \\
2.08\end{array}$ & $\begin{array}{c}106.34 \pm \\
10.38\end{array}$ & $\begin{array}{c}20.52 \pm \\
0.90\end{array}$ & $\begin{array}{c}0.53 \pm \\
0.10\end{array}$ & $\begin{array}{c}0.32 \pm \\
0.04\end{array}$ & $\begin{array}{c}36.49 \pm \\
2.20\end{array}$ \\
\hline & $\begin{array}{c}20-30 \\
\mathrm{~cm}\end{array}$ & $\begin{array}{c}98.85 \pm \\
3.83\end{array}$ & $\begin{array}{c}27.64 \pm \\
0.56\end{array}$ & $\begin{array}{c}39.26 \pm \\
2.08\end{array}$ & $\begin{array}{c}97.24 \pm \\
2.00\end{array}$ & $\begin{array}{c}21.03 \pm \\
1.75\end{array}$ & $\begin{array}{c}0.58 \pm \\
0.04\end{array}$ & $\begin{array}{c}0.28 \pm \\
0.02\end{array}$ & $\begin{array}{c}37.86 \pm \\
1.33\end{array}$ \\
\hline \multirow{3}{*}{$\mathrm{S} 11$} & $0-10 \mathrm{~cm}$ & $\begin{array}{c}91.48 \pm \\
6.88\end{array}$ & $\begin{array}{c}25.98 \pm \\
0.29\end{array}$ & $\begin{array}{c}32.51 \pm \\
0.89\end{array}$ & $\begin{array}{c}128.32 \pm \\
32.57\end{array}$ & $\begin{array}{c}19.82 \pm \\
1.06\end{array}$ & $\begin{array}{c}0.50 \pm \\
0.05\end{array}$ & $\begin{array}{c}2.84 \pm \\
2.42\end{array}$ & $\begin{array}{c}36.42 \pm \\
2.21\end{array}$ \\
\hline & $\begin{array}{c}10-20 \\
\mathrm{~cm}\end{array}$ & $\begin{array}{c}98.17 \pm \\
4.36\end{array}$ & $\begin{array}{c}27.82 \pm \\
1.24\end{array}$ & $\begin{array}{c}36.72 \pm \\
2.38\end{array}$ & $\begin{array}{c}124.35 \pm \\
27.78\end{array}$ & $\begin{array}{c}49.26 \pm \\
28.19\end{array}$ & $\begin{array}{c}1.01 \pm \\
0.37\end{array}$ & $\begin{array}{c}0.78 \pm \\
0.42\end{array}$ & $\begin{array}{c}47.11 \pm \\
10.30\end{array}$ \\
\hline & $\begin{array}{c}20-30 \\
\mathrm{~cm}\end{array}$ & $\begin{array}{c}159.26 \pm \\
56.12\end{array}$ & $\begin{array}{c}34.98 \pm \\
8.21\end{array}$ & $\begin{array}{c}37.11 \pm \\
2.29\end{array}$ & $\begin{array}{c}110.31 \pm \\
12.89\end{array}$ & $\begin{array}{c}22.35 \pm \\
0.87\end{array}$ & $\begin{array}{c}0.92 \pm \\
0.16\end{array}$ & $\begin{array}{c}0.49 \pm \\
0.19\end{array}$ & $\begin{array}{c}40.52 \pm \\
0.47\end{array}$ \\
\hline \multirow{3}{*}{ S12 } & $0-10 \mathrm{~cm}$ & $\begin{array}{c}90.15 \pm \\
9.27\end{array}$ & $\begin{array}{c}30.10 \pm \\
5.03\end{array}$ & $\begin{array}{c}32.75 \pm \\
1.79\end{array}$ & $\begin{array}{c}104.86 \pm \\
2.56\end{array}$ & $\begin{array}{c}17.86 \pm \\
1.80\end{array}$ & $\begin{array}{c}0.49 \pm \\
0.03\end{array}$ & $\begin{array}{c}0.31 \pm \\
0.04\end{array}$ & $\begin{array}{c}36.01 \pm \\
3.13\end{array}$ \\
\hline & $\begin{array}{c}10-20 \\
\mathrm{~cm}\end{array}$ & $\begin{array}{c}107.01 \pm \\
2.19\end{array}$ & $\begin{array}{c}32.14 \pm \\
2.67\end{array}$ & $\begin{array}{c}37.97 \pm \\
1.16\end{array}$ & $\begin{array}{c}108.61 \pm \\
5.50\end{array}$ & $\begin{array}{c}19.92 \pm \\
0.44\end{array}$ & $\begin{array}{c}0.71 \pm \\
0.06\end{array}$ & $\begin{array}{c}0.35 \pm \\
0.04\end{array}$ & $\begin{array}{c}44.19 \pm \\
0.12\end{array}$ \\
\hline & $\begin{array}{c}20-30 \\
\mathrm{~cm}\end{array}$ & $\begin{array}{c}103.50 \pm \\
3.03\end{array}$ & $\begin{array}{c}32.20 \pm \\
0.53\end{array}$ & $\begin{array}{c}40.53 \pm \\
0.90\end{array}$ & $\begin{array}{c}115.91 \pm \\
3.32\end{array}$ & $\begin{array}{c}23.56 \pm \\
0.64\end{array}$ & $\begin{array}{c}0.83 \pm \\
0.01\end{array}$ & $\begin{array}{c}0.32 \pm \\
0.03\end{array}$ & $\begin{array}{c}46.89 \pm \\
0.93\end{array}$ \\
\hline \multirow{3}{*}{ S13 } & $0-10 \mathrm{~cm}$ & $\begin{array}{c}95.03 \pm \\
6.05\end{array}$ & $\begin{array}{c}30.16 \pm \\
2.46\end{array}$ & $\begin{array}{c}44.76 \pm \\
3.44\end{array}$ & $\begin{array}{c}118.77 \pm \\
6.86\end{array}$ & $\begin{array}{c}23.11 \pm \\
1.50\end{array}$ & $\begin{array}{c}0.60 \pm \\
0.03\end{array}$ & $\begin{array}{c}0.12 \pm \\
0.02\end{array}$ & $\begin{array}{c}40.40 \pm \\
1.88\end{array}$ \\
\hline & $\begin{array}{c}10-20 \\
\mathrm{~cm}\end{array}$ & $\begin{array}{c}99.64 \pm \\
6.54\end{array}$ & $\begin{array}{c}32.35 \pm \\
0.37\end{array}$ & $\begin{array}{c}43.79 \pm \\
1.12\end{array}$ & $\begin{array}{c}115.31 \pm \\
14.37\end{array}$ & $\begin{array}{c}22.60 \pm \\
0.39\end{array}$ & $\begin{array}{c}0.80 \pm \\
0.08\end{array}$ & $\begin{array}{c}0.12 \pm \\
0.02\end{array}$ & $\begin{array}{c}44.69 \pm \\
0.87\end{array}$ \\
\hline & $\begin{array}{c}20-30 \\
\mathrm{~cm}\end{array}$ & $\begin{array}{c}93.22 \pm \\
5.56\end{array}$ & $\begin{array}{c}29.44 \pm \\
2.14\end{array}$ & $\begin{array}{c}40.58 \pm \\
0.71\end{array}$ & $\begin{array}{c}105.90 \pm \\
1.81\end{array}$ & $\begin{array}{c}22.60 \pm \\
0.77\end{array}$ & $\begin{array}{c}0.66 \pm \\
0.02\end{array}$ & $\begin{array}{c}0.11 \pm \\
0.01\end{array}$ & $\begin{array}{c}38.80 \pm \\
1.92\end{array}$ \\
\hline Average & & $\begin{array}{c}99.44 \pm \\
2.59\end{array}$ & $\begin{array}{c}29.25 \pm \\
0.47\end{array}$ & $\begin{array}{c}38.99 \pm \\
0.75\end{array}$ & $\begin{array}{c}113.96 \pm \\
4.55\end{array}$ & $\begin{array}{c}31.02 \pm \\
4.44\end{array}$ & $\begin{array}{c}1.23 \pm \\
0.24\end{array}$ & $\begin{array}{c}0.38 \pm \\
0.11\end{array}$ & $\begin{array}{c}45.36 \pm \\
2.55\end{array}$ \\
\hline
\end{tabular}

\subsection{Lower Intertidal Zone}

The average concentration of the investigated trace elements showed the decreasing order: $\mathrm{Zn}>$ $\mathrm{Cr}>\mathrm{Pb}>\mathrm{Cu}>\mathrm{Ni}>\mathrm{As}>\mathrm{Cd}>\mathrm{Hg}$, which is different to those in the upper and mid intertidal zones. The soil-borne trace elements were only slightly variable horizontally. No clear pattern of vertical variation in the soil-borne trace elements was observed. An unusually high concentration of Cd was observed for the surface soil layer at Location S17 (Table 5). 
Table 5. Mangrove soil-borne trace elements $(\mathrm{mg} / \mathrm{kg})$ in the lower intertidal zone.

\begin{tabular}{|c|c|c|c|c|c|c|c|c|c|}
\hline Sample & Depth & $\mathrm{Cr}$ & $\mathbf{N i}$ & $\mathrm{Cu}$ & Zn & As & $\mathrm{Cd}$ & $\mathrm{Hg}$ & $\mathrm{Pb}$ \\
\hline \multirow{3}{*}{ S14 } & $0-10 \mathrm{~cm}$ & $\begin{array}{c}79.60 \pm \\
0.74\end{array}$ & $\begin{array}{c}24.20 \pm \\
0.56\end{array}$ & $\begin{array}{c}34.20 \pm \\
4.12\end{array}$ & $\begin{array}{c}109.30 \pm \\
9.33\end{array}$ & $\begin{array}{c}39.79 \pm \\
22.99\end{array}$ & $\begin{array}{c}4.02 \pm \\
3.70\end{array}$ & $\begin{array}{c}0.07 \pm \\
0.00\end{array}$ & $\begin{array}{c}33.68 \pm \\
3.22\end{array}$ \\
\hline & $\begin{array}{c}10-20 \\
\mathrm{~cm}\end{array}$ & $\begin{array}{c}82.70 \pm \\
7.25\end{array}$ & $\begin{array}{c}27.90 \pm \\
0.37\end{array}$ & $\begin{array}{c}36.20 \pm \\
3.35\end{array}$ & $\begin{array}{c}101.99 \pm \\
7.16\end{array}$ & $\begin{array}{c}18.51 \pm \\
0.97\end{array}$ & $\begin{array}{c}0.42 \pm \\
0.03\end{array}$ & $\begin{array}{c}0.07 \pm \\
0.01\end{array}$ & $\begin{array}{c}33.69 \pm \\
2.40\end{array}$ \\
\hline & $\begin{array}{c}20-30 \\
\mathrm{~cm}\end{array}$ & $\begin{array}{c}107.00 \pm \\
18.10\end{array}$ & $\begin{array}{c}32.80 \pm \\
3.68\end{array}$ & $\begin{array}{c}45.20 \pm \\
6.78\end{array}$ & $\begin{array}{c}138.67 \pm \\
21.62\end{array}$ & $\begin{array}{c}40.15 \pm \\
15.17\end{array}$ & $\begin{array}{c}1.54 \pm \\
0.88\end{array}$ & $\begin{array}{c}0.10 \pm \\
0.02\end{array}$ & $\begin{array}{c}51.64 \pm \\
7.93\end{array}$ \\
\hline \multirow{3}{*}{ S15 } & $0-10 \mathrm{~cm}$ & $\begin{array}{c}81.60 \pm \\
6.28\end{array}$ & $\begin{array}{c}27.40 \pm \\
0.23\end{array}$ & $\begin{array}{c}38.90 \pm \\
0.93\end{array}$ & $\begin{array}{c}65.74 \pm \\
47.90\end{array}$ & $\begin{array}{c}12.66 \pm \\
7.04\end{array}$ & $\begin{array}{c}2.26 \pm \\
1.90\end{array}$ & $\begin{array}{c}0.08 \pm \\
0.00\end{array}$ & $\begin{array}{c}34.33 \pm \\
3.96\end{array}$ \\
\hline & $\begin{array}{c}10-20 \\
\mathrm{~cm}\end{array}$ & $\begin{array}{c}106.00 \pm \\
24.5\end{array}$ & $\begin{array}{c}34.60 \pm \\
6.24\end{array}$ & $\begin{array}{c}39.60 \pm \\
2.98\end{array}$ & $\begin{array}{c}112.05 \pm \\
4.54\end{array}$ & $\begin{array}{c}20.55 \pm \\
1.71\end{array}$ & $\begin{array}{c}0.47 \pm \\
0.05\end{array}$ & $\begin{array}{c}0.08 \pm \\
0.01\end{array}$ & $\begin{array}{c}32.75 \pm \\
2.77\end{array}$ \\
\hline & $\begin{array}{c}20-30 \\
\mathrm{~cm}\end{array}$ & $\begin{array}{c}99.30 \pm \\
9.77\end{array}$ & $\begin{array}{c}31.90 \pm \\
1.81\end{array}$ & $\begin{array}{c}53.00 \pm \\
9.39\end{array}$ & $\begin{array}{c}312.17 \pm \\
173.62\end{array}$ & $\begin{array}{c}17.74 \pm \\
5.84\end{array}$ & $\begin{array}{c}1.08 \pm \\
0.43\end{array}$ & $\begin{array}{c}0.10 \pm \\
0.02\end{array}$ & $\begin{array}{c}45.59 \pm \\
4.72\end{array}$ \\
\hline \multirow{3}{*}{ S16 } & $0-10 \mathrm{~cm}$ & $\begin{array}{c}83.50 \pm \\
13.20\end{array}$ & $\begin{array}{c}30.60 \pm \\
0.09\end{array}$ & $\begin{array}{c}43.50 \pm \\
2.27\end{array}$ & $\begin{array}{c}119.37 \pm \\
7.94\end{array}$ & $\begin{array}{c}21.71 \pm \\
0.90\end{array}$ & $\begin{array}{c}0.51 \pm \\
0.10\end{array}$ & $\begin{array}{c}0.10 \pm \\
0.00\end{array}$ & $\begin{array}{c}34.98 \pm \\
4.71\end{array}$ \\
\hline & $\begin{array}{c}10-20 \\
\mathrm{~cm}\end{array}$ & $\begin{array}{c}151.00 \pm \\
68.10\end{array}$ & $\begin{array}{c}48.30 \pm \\
17.20\end{array}$ & $\begin{array}{c}44.70 \pm \\
5.16\end{array}$ & $\begin{array}{c}126.34 \pm \\
8.33\end{array}$ & $\begin{array}{c}21.39 \pm \\
0.72\end{array}$ & $\begin{array}{c}0.59 \pm \\
0.04\end{array}$ & $\begin{array}{c}0.08 \pm \\
0.01\end{array}$ & $\begin{array}{c}38.28 \pm \\
2.74\end{array}$ \\
\hline & $\begin{array}{c}20-30 \\
\mathrm{~cm}\end{array}$ & $\begin{array}{c}91.30 \pm \\
1.68\end{array}$ & $\begin{array}{c}30.90 \pm \\
0.74\end{array}$ & $\begin{array}{c}60.80 \pm \\
12.00\end{array}$ & $\begin{array}{c}190.22 \pm \\
44.09\end{array}$ & $\begin{array}{c}21.79 \pm \\
1.81\end{array}$ & $\begin{array}{c}0.62 \pm \\
0.04\end{array}$ & $\begin{array}{c}0.10 \pm \\
0.02\end{array}$ & $\begin{array}{c}39.54 \pm \\
1.65\end{array}$ \\
\hline \multirow{3}{*}{ S17 } & $0-10 \mathrm{~cm}$ & $\begin{array}{c}91.30 \pm \\
2.97 \\
\end{array}$ & $\begin{array}{c}32.00 \pm \\
1.31 \\
\end{array}$ & $\begin{array}{c}39.20 \pm \\
2.07 \\
\end{array}$ & $\begin{array}{c}120.63 \pm \\
15.90\end{array}$ & $\begin{array}{c}40.38 \pm \\
19.91 \\
\end{array}$ & $\begin{array}{c}0.87 \pm \\
0.36\end{array}$ & $\begin{array}{c}0.09 \pm \\
0.03\end{array}$ & $\begin{array}{c}45.68 \pm \\
9.21 \\
\end{array}$ \\
\hline & $\begin{array}{c}10-20 \\
\mathrm{~cm}\end{array}$ & $\begin{array}{c}95.30 \pm \\
3.03\end{array}$ & $\begin{array}{c}30.00 \pm \\
0.42\end{array}$ & $\begin{array}{c}42.30 \pm \\
1.64\end{array}$ & $\begin{array}{c}113.64 \pm \\
5.59\end{array}$ & $\begin{array}{c}21.69 \pm \\
0.85\end{array}$ & $\begin{array}{c}0.54 \pm \\
0.04\end{array}$ & $\begin{array}{c}0.08 \pm \\
0.02\end{array}$ & $\begin{array}{c}41.54 \pm \\
1.49\end{array}$ \\
\hline & $\begin{array}{c}20-30 \\
\mathrm{~cm}\end{array}$ & $\begin{array}{c}95.80 \pm \\
5.41\end{array}$ & $\begin{array}{c}32.70 \pm \\
3.11\end{array}$ & $\begin{array}{c}48.70 \pm \\
1.76\end{array}$ & $\begin{array}{c}131.08 \pm \\
2.42\end{array}$ & $\begin{array}{c}22.61 \pm \\
1.31\end{array}$ & $\begin{array}{c}0.65 \pm \\
0.06\end{array}$ & $\begin{array}{c}0.08 \pm \\
0.02\end{array}$ & $\begin{array}{c}42.34 \pm \\
3.19\end{array}$ \\
\hline Average & & $\begin{array}{c}101.55 \pm \\
2.74\end{array}$ & $\begin{array}{c}30.37 \pm \\
0.75\end{array}$ & $\begin{array}{c}40.76 \pm \\
1.08\end{array}$ & $\begin{array}{c}124.84 \pm \\
8.93\end{array}$ & $\begin{array}{c}26.26 \pm \\
2.16\end{array}$ & $\begin{array}{c}0.94 \pm \\
0.13\end{array}$ & $\begin{array}{c}0.28 \pm \\
0.08\end{array}$ & $\begin{array}{c}43.35 \pm \\
1.40\end{array}$ \\
\hline
\end{tabular}

\section{Discussion}

The sedimentation rate in the tidal zone of Qi'ao Island was approximately $1.68 \mathrm{~cm} /$ year [22]. Therefore, the mangrove soils $(0-30 \mathrm{~cm})$ investigated in this study were mostly derived from sediments deposited during the past two decades. Due to the very small local catchment size, it was unlikely that soil erosion from the hill slopes contributed significantly to the tidal sediments. Most of the sediments present in the tidal zone should have been deposited from the suspended materials discharged into the Pearl River estuary from the Pearl River. It is clear from the results that spatial variation in the investigated soil-borne trace elements was minor, suggesting that the whole intertidal zone received relatively constant amounts of trace elements from the Pearl River catchment in the past two decades. This can be used to indicate that the trace element loadings in the Pearl River distributaries were generally stable during the recent two decades.

Chromium was not related to any other investigated trace elements (Table 6). This appears to suggest that this trace element was not largely derived from the same water pollution sources as the other seven investigated trace elements. The major water pollution sources of $\mathrm{Cr}$ include chrome plating, leather tanning, and textile industries [23-25], which do not release substantial amounts of other trace elements. 
Table 6. Correlation matrix showing the correlation coefficients between the investigated trace elements.

\begin{tabular}{|c|c|c|c|c|c|c|c|c|}
\hline & $\mathrm{Cr}$ & $\mathrm{Ni}$ & $\mathrm{Cu}$ & $\mathrm{Zn}$ & As & $\mathrm{Cd}$ & $\mathrm{Hg}$ & $\mathrm{Pb}$ \\
\hline $\mathrm{Cr}$ & 1 & & & & & & & \\
\hline $\mathrm{Ni}$ & 0.129 & 1 & & & & & & \\
\hline $\mathrm{Cu}$ & -0.163 & $0.549 * *$ & 1 & & & & & \\
\hline $\mathrm{Zn}$ & -0.317 & 0.007 & $0.459 *$ & 1 & & & & \\
\hline As & -0.382 & -0.049 & 0.389 & $0.965^{* *}$ & 1 & & & \\
\hline $\mathrm{Cd}$ & -0.082 & -0.163 & 0.396 & $0.550^{* *}$ & $0.495^{*}$ & 1 & & \\
\hline $\mathrm{Hg}$ & 0.316 & -0.394 & -0.079 & 0.017 & 0.010 & 0.270 & 1 & \\
\hline $\mathrm{Pb}$ & 0.171 & -0.267 & -0.089 & $0.450 *$ & 0.384 & 0.360 & -0.054 & 1 \\
\hline
\end{tabular}

Mercury was also not related to any other trace elements (Table 6). Mining is a major source of mercury in many places [26]. However, $\mathrm{Hg}$ discharge related to mining activities is always accompanied with discharges of heavy metals and metalloids [27]. Therefore, Hg discharged into Pearl River was likely to be due to other sources, such as pulp and paper industries [28].

There was a close relationship between $\mathrm{Pb}$ and $\mathrm{Zn}$ in the investigated mangrove soils (Table 6). This can be explained by the close association between lead sulfide and zinc sulfides in lead-zinc deposits [29]. Mining and smelting of lead-zinc ores result in simultaneous discharge of both $\mathrm{Pb}$ and $\mathrm{Zn}$.

The close relationship between $\mathrm{Ni}$ and $\mathrm{Cu}$ (Table 6) could be attributed to their concurrent presence in nickel-copper sulfide ores [30]. The sources of nickel include nickel plating, non-Ni ore mining, burning of coal and oil, nickel-cadmium batteries, sewage, phosphate fertilizers, and pesticides, which also involve other trace elements [31]. The fact that $\mathrm{Ni}$ was only closely related to $\mathrm{Cu}$ but not to other trace elements suggests that these are not major contributors to the Ni loading in the Pearl River system.

It is interesting to note that $\mathrm{Zn}$ was not only closely related to $\mathrm{Pb}$ but also to $\mathrm{Cu}, \mathrm{Cd}$, and $\mathrm{As}$ (Table 6). $\mathrm{Zn}$ discharge can be caused by a wide range of economic activities, including mining, smelting, coal burning, manufacturing, and the use of pesticides and fungicides [32-34], which frequently involve other trace elements.

It is also interesting to note that there is a trend showing that the concentration of $\mathrm{Cd}$ and $\mathrm{Hg}$ decreased from the upper intertidal zone to the lower intertidal zone. This suggests that these two elements tended to be retained in the upper part of the tidal zone and their seaward transport was relatively weak. The extremely high concentration of $\mathrm{Cd}$ and $\mathrm{Hg}$ in the surface soil layer at some locations was accompanied by a large standard deviation, indicating the significant variation among the three replicate samples collected at the same location. This can be attributed to unusual contamination by local sources at certain isolated spots. Further investigation is needed to understand the causes of these observations.

The background concentrations of $\mathrm{Cr}, \mathrm{Ni}, \mathrm{Cu}, \mathrm{Zn}, \mathrm{As}, \mathrm{Cd}, \mathrm{Hg}$, and $\mathrm{Pb}$ were 50.5, 14.4, 17, 47.3, 8.9, $0.05,0.078$, and $36 \mathrm{mg} / \mathrm{kg}$, respectively. It can be seen from Figure 2 that the concentrations of the eight investigated soil-borne trace elements were all greater than the respective background concentration of each trace element. 

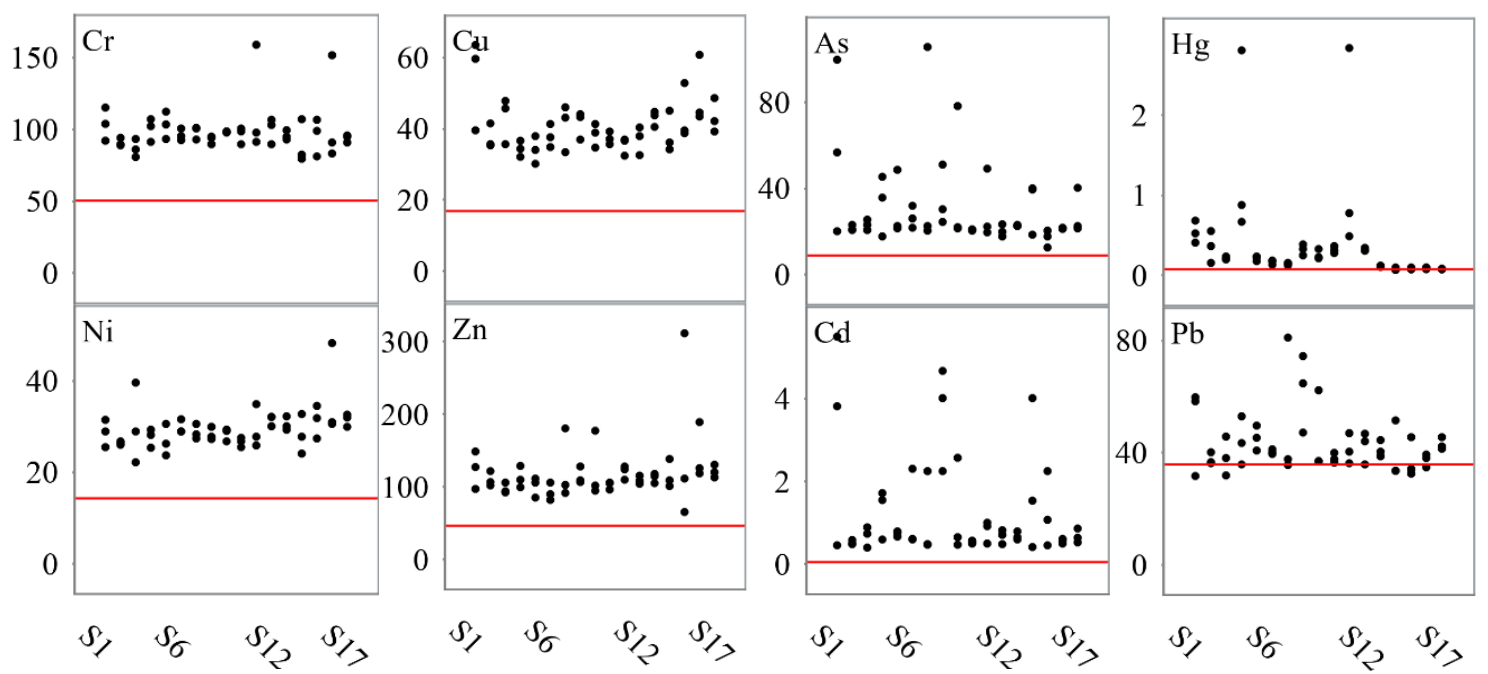

Figure 2. Concentration of the investigated soil-borne trace elements $(\mathrm{Cr}, \mathrm{Cu}, \mathrm{As}, \mathrm{Hg}, \mathrm{Ni}, \mathrm{Zn}, \mathrm{Cd}$ and $\mathrm{Pb}$ ) compared to their respective background values.

The INPI of the surface soils at the 17 sampling locations ranged from 7.53 to 48.42 , values which all fall within the highest pollution category (Category 5), i.e., heavy pollution (Table 7).

Table 7. The integrated Nemerow pollution index (INPI) of the surface soils at the 17 sampling locations.

\begin{tabular}{ccc}
\hline Sampling Location & INPI & Degree of Pollution \\
\hline S1 & 12.74 & Heavy pollution \\
S2 & 11.41 & Heavy pollution \\
\hline S3 & 14.52 & Heavy pollution \\
S4 & 16.38 & Heavy pollution \\
\hline S5 & 14.29 & Heavy pollution \\
S6 & 48.42 & Heavy pollution \\
\hline S7 & 16.45 & Heavy pollution \\
S8 & 7.53 & Heavy pollution \\
\hline S9 & 11.72 & Heavy pollution \\
S10 & 9.31 & Heavy pollution \\
\hline S11 & 9.56 & Heavy pollution \\
S12 & 9.90 & Heavy pollution \\
S13 & 17.69 & Heavy pollution \\
\hline S17 & 22.70 & Heavy pollution \\
S18 & 15.18 & Heavy pollution \\
\hline S19 & 8.02 & Heavy pollution \\
S20 & 9.35 & Heavy pollution \\
\hline
\end{tabular}

Using the ecological risk assessment method of Hakanson [19], among the 17 sampling locations, six locations had an ERI greater than 300 and 12 locations had an ERI greater than 600 (Figure 3), indicating that most of the investigated locations were at high or very high ecological risk. 


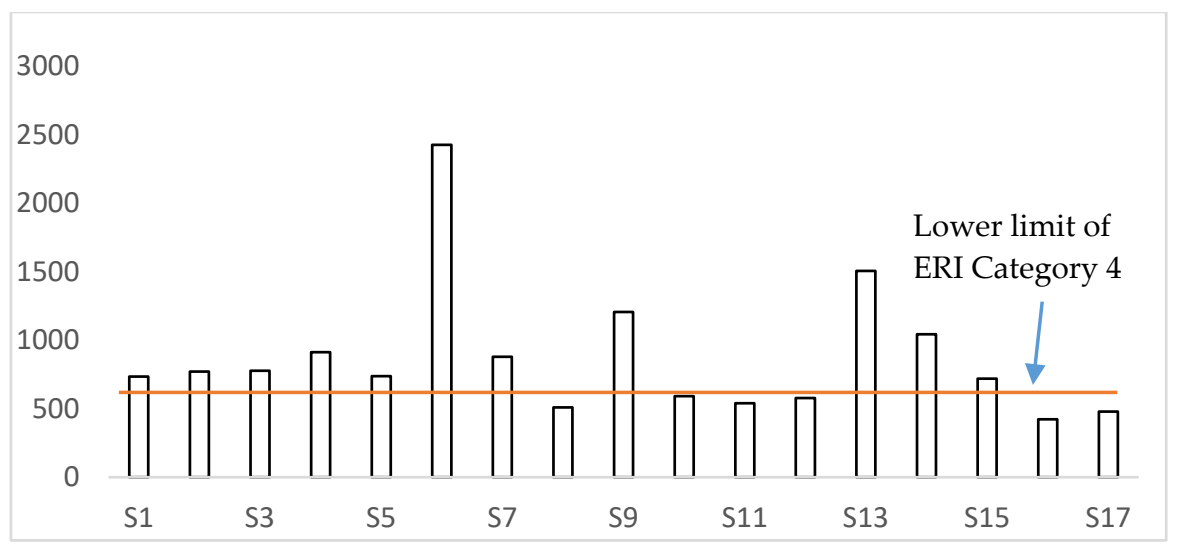

Figure 3. Comparison of ERI among the 17 sampling locations in the investigated area.

By comparison with some other mangrove wetlands around the world, the concentration of soil-borne $\mathrm{Cr}, \mathrm{Zn} \mathrm{As}, \mathrm{Cd}$, and $\mathrm{Hg}$ was higher at this study site than in the majority of the reference sites (Table 8). Qi'ao Island is the island closest to the mouths of two major active distributaries of the Pearl River. Therefore, the mangrove tidal zone was directly exposed to the sediment-borne trace elements discharged from the Pearl River. Although a firm conclusion cannot be drawn from the data obtained at this study site alone, the special location of Qi'ao Island does allow some level of confidence to suggest that Pearl River is amongst the heavily contaminated rivers around the world.

Table 8. Levels of heavy metals in mangrove wetland soils.

\begin{tabular}{ccccccccc}
\hline Study Area & $\mathbf{C r}$ & $\mathbf{N i}$ & $\mathbf{C u}$ & $\mathbf{Z n}$ & $\mathbf{A s}$ & $\mathbf{C d}$ & $\mathbf{H g}$ & $\mathbf{P b}$ \\
\hline Sanya, China [35] & 61.5 & - & 21.0 & 126 & 14.3 & 0.09 & - & 42.3 \\
\hline Luoyang Bridge, China [35] & 18.6 & - & 34.0 & 106 & 5.31 & 0.06 & - & 167 \\
\hline Dongzhai Harbor, China [36] & 75.8 & 31.0 & 19.6 & 44.8 & 8.67 & 0.62 & - & 20.7 \\
\hline Yingluo Harbor, China [37] & 56.7 & - & 7.76 & 31.9 & - & 0.11 & 0.04 & 14.2 \\
\hline Shenzhen Bay, China [7] & 96.2 & 44.6 & 88.8 & 358 & 172 & 0.94 & 0.14 & 72 \\
\hline Zhangjiang River Estuary, China [38] & - & - & 21.04 & - & - & 0.33 & 0.03 & 63.2 \\
\hline Sunderban mangrove, India [39] & 41.8 & 47.4 & 60.06 & 88.3 & - & 0.48 & 0.24 & 52.9 \\
\hline Guanabara Bay, Brazil [40] & 42.4 & - & 98.6 & 483 & 1.32 & 1.32 & - & 160 \\
\hline Kottuli, India [41] & 0.26 & 0.38 & 69.3 & 384 & - & 0.03 & - & 6.91 \\
\hline Hong Kong, China [11] & 40.0 & 3.00 & 240 & 40.0 & - & 3.00 & - & 80 \\
\hline Pichavaram, India [42] & 530 & 253 & 150 & 108 & - & 34.5 & - & 133 \\
\hline Brisbane River, Australia [43] & $7.6-116$ & $2.4-57.6$ & $3.1-34.1$ & $40-144$ & $0-13.0$ & $0-2.0$ & - & $7.7-84.7$ \\
\hline This Study & 99.5 & 29.2 & 40.8 & 116 & 30.0 & 1.16 & 0.41 & 44.0 \\
\hline
\end{tabular}

\section{Conclusions}

The mangrove soils of Qi'ao Island were heavily contaminated by trace elements transported from the Pearl River. These soil-borne trace elements pose a high risk to the mangrove ecosystem. The whole intertidal zone received relatively constant amounts of trace elements from the Pearl River catchment in the past two decades. Zn showed common sources with many other trace elements while some trace elements were not closely related to each other. The findings obtained from this study have implications for understanding the terrestrial inputs of trace elements into part of the Pearl River estuary. Although a firm conclusion cannot be drawn from the data obtained at this study site alone, the special location of Qi'ao Island does allow some level of confidence to suggest that Pearl River is amongst the heavily contaminated rivers in the world. This will provide useful information for 
decision-makers to better control the discharge of trace elements from the catchment area and manage the aquatic ecosystems in the Pearl River Estuary.

Author Contributions: Conceptualization, S.X. and A.N.; methodology, A.N., J.M.; investigation, A.N., J.M., and Y.G.; resources, S.X.; data curation, A.N. and Y.G.; writing-original draft preparation, A.N.; writing-review and editing, C.L.; visualization, Y.G. and A.N.; supervision, S.X. and C.L.; project administration, S.X.; funding acquisition, S.X. All authors have read and agreed to the published version of the manuscript.

Funding: This research was funded by the National Natural Science Foundation of China, grant number 41877411.

Conflicts of Interest: The authors declare no conflicts of interest.

\section{References}

1. Bayen, S. Occurrence, bioavailability and toxic effects of trace metals and organic contaminants in mangrove ecosystems: A review. Environ. Int. 2012, 48, 84-101. [CrossRef] [PubMed]

2. Kumar, A.; Ramanathan, A.L.; Prasad, M.B.K.; Datta, D.; Kumar, M.; Sappal, S.M. Distribution, enrichment, and potential toxicity of trace metals in the surface sediments of Sundarban mangrove ecosystem, Bangladesh: A baseline study before Sundarban oil spill of December, 2014. Environn. Sci. Pollut. R. 2016, 9, 8985-8999. [CrossRef] [PubMed]

3. Machado, W.; Silva-Filho, E.V.; Oliveira, R.R.; Lacerda, L.D. Trace metal retention in mangrove ecosystems in Guanabara Bay, SE Brazil. Mar. Pollut. Bull. 2002, 11, 1277-1280. [CrossRef]

4. Soto-Jimenez, M.F.; Paez-Osuna, F. Distribution and normalization of heavy metal concentrations in mangrove and lagoonal sediments from Mazatlan Harbor (SE Gulf of California). Estuar. Coast. Shelf Sci. 2001, 3, 259-274. [CrossRef]

5. Ahmed, A.; Ohlson, M.; Hoque, S.; Moula, M.G. Chemical composition of leaves of a mangrove tree (Sonneratia apetala buch.-ham.) And their correlation with some soil variables. Bangladesh J. Bot. 2010, 1, 61-69. [CrossRef]

6. Naidoo, G.; Hiralal, T.; Naidoo, Y. Ecophysiological responses of the mangrove Avicennia marina to trace metal contamination. Flora 2014, 1, 63-72. [CrossRef]

7. He, B.; Li, R.; Chai, M.; Qiu, G. Threat of heavy metal contamination in eight mangrove plants from the Futian mangrove forest, China. Environ. Geochem. Health 2014, 3, 467-476. [CrossRef]

8. Liu, Y.; Tam, N.F.Y.; Yang, J.X.; Pi, N.; Wong, M.H.; Ye, Z.H. Mixed heavy metals tolerance and radial oxygen loss in mangrove seedlings. Mar. Pollut. Bull. 2009, 12, 1843-1849. [CrossRef]

9. Hu, Z. Studies on the Discharging and Distribution of Heavy Metal Pollution in the Pearl River Delta. Ph.D. Thesis, Graduate School of the Chinese Academy of Sciences Guangzhou Institute of Geochemistry, Guangzhou, China, 2004.

10. Ministry of Ecology and Environment of People's Republic of China. Bulletin of the State of the Environment of China; Ministry of Ecology and Environment of People's Republic of China: Beijing, China, 2011.

11. Tam, N.F.Y.; Wong, Y. Spatial variation of heavy metals in surface sediments of Hong Kong mangrove swamps. Environ. Pollut. 2000, 2, 195-205. [CrossRef]

12. Liang, Y.; Wong, M. Spatial and temporal organic and heavy metal pollution at Mai Po Marshes Nature Reserve, Hong Kong. Chemosphere 2003, 52, 1647-1658. [CrossRef]

13. Food and Agriculture Organization of the United Nations. World Reference Base for Soil Resources-WBR, 2nd ed.; Food and Agriculture Organization of the United Nations: Roma, Italy, 2006.

14. Wei, G.; Baowen, L.; Fengying, Q.; Xinghua, G.; Jing, H.; Guanghong, T.; Xiongbang, Y. A Preliminary study on controlling the invasive species spartina alterniflora using Sonneratia apetala. Forest Res. 2009, 4, 603-607. [CrossRef]

15. Guanghong, T.; Mingyan, D.; Xiongbang, Y.; Yanjun, S.; Wenbo, L.; Zhen, L.; Jiajun, G. Characteristics of sarcosperma laurinum community and species diversity from Qi-ao island in Zhuhai city, Guangdong province. Plant Sci. J. 2013, 5, 461-466. [CrossRef]

16. Yijie, T.; Zhanqiang, F.; Yangting, Z.; Zaiwang, Z.; Kang, C.; Dong, A.; Xiongbang, Y.; Baowen, L. Succession of macrofauna communities in wetlands of Sonneratia apetala artificial mangroves during different ecological restoration stages. Acta Ecol. Sin. 2012, 10, 3160-3169. [CrossRef] 
17. Yang, Z.; Lu, W.; Long, Y.; Bao, X.; Yang, Q. Assessment of heavy metals contamination in urban topsoil from Changchun City, China. J. Geochem. Explor. 2011, 108, 27-38. [CrossRef]

18. Hou, X.; Huang, J.; Liu, A. Heavy metals pollution and it's assessment in the wetlands of min river estuary in Fujian province. J. Agro-Environ. Sci. 2009, 11, 2302-2306.

19. Hakanson, L. An ecological risk index for aquatic pollution control, a sediment-ecological approach. Water Res. 1980, 14, 975-1001. [CrossRef]

20. Guo, W.; Liu, X.; Liu, Z.; Li, G. Pollution and potential ecological risk evaluation of heavy metals in the sediments around dongjiang harbor, Tianjin. Procedia Environ. Sci. 2010, 2, 729-736. [CrossRef]

21. Xu, Q.; Ni, S.; Tuo, X.; Zhang, C. Calculation of heavy metals' toxicity coefficient in the evaluation of potential ecological risk index. Environ. Sci. Technol. 2008, 2, 112-115. [CrossRef]

22. Ruili, L.; Minwei, C.; Guoyu, Q.; Bei, H. Mercury and copper accumulation during last fifty years and their potential ecological risk assessment in sediment of mangrove wetland of shenzhen, China. Chin. J. Environ Sci. 2012, 12, 4276-4283.

23. Avudainayagam, S.; Megharaj, M.; Owens, G.; Kookana, R.S.; Chittleborough, D.; Naidu, R. Chemistry of chromium in soils with emphasis on tannery waste sites. Rev. Environ. Contam. Toxicol. 2003, 178, 53-91. [CrossRef]

24. Zhao, Y.; Guo, H.; Sun, Z.; Shi, X. Wu, K.N. Principle component analyses based on soil knowledge as a tool to indicate origin of heavy metals in soils. Sci. Geogr. Sin. 2008, 1, 45-50. [CrossRef]

25. Sun, H.; Bi, T.; Guo, Y.; Yuan, Y.; Chai, M.; Guo, Z. Source apportionment analysis of trace metal contamination in soils of Guangdong province, China. Acta Sci. Circumstantiae 2018, 2, 704-714.

26. Zhang, H.; Chen, J.; Zhu, L.; Yang, G.; Li, D. Anthropogenic mercury enrichment factors and contributions in soils of guangdong province, South China. J. Geochem. Explor. 2014, 144, 312-319. [CrossRef]

27. David, G.; Jiming, H.; Ye, W.; Jingkun, J.; Melissa, C.; Hezhong, T.; Xinbin, F. Anthropogenic mercury emissions in China. Atmos. Environ. 2005, 40, 7789-7806. [CrossRef]

28. Zhang, Q.; Liu, X.; Zhou, J.; Liu, X. Contents and risk assessment of heavy metal sediments in Guangdong coastal areas. China Environ. Sci. 2018, 12, 4653-4660. [CrossRef]

29. Newton, L. Pollution of the rivers of west wales by lead and zinc mine effluent. Ann. Appl. Biol. 2008, 1, 1-11. [CrossRef]

30. Xueshu, Z.; Franco, P.; Dexian, Q.; Zhuguo, F.; Guanliang, L.; Hong, N. Baimazhai, Yunnan province, China: A hydrothermally modified magmatic nickel-copper-pge sulfide deposit. Int. Geol. Rev. 2006, 8, 725-741. [CrossRef]

31. Hutchinson, T.C.; Whitby, L.M. Heavy-metal pollution in the sudbury mining and smelting region of canada, I. soil and vegetation contamination by nickel, copper, and other metals. Environ. Conserv. 1974, 2, 123-132. [CrossRef]

32. Hashem, M.A.; Nur-A-Tomal, M.S.; Mondal, N.R.; Rahman, M.A. Hair burning and liming in tanneries is a source of pollution by arsenic, lead, zinc, manganese and iron. Environ. Chem. Lett. 2017, 3, 501-506. [CrossRef]

33. Urszula, A.K.; Dariusz, C. Groundwater hydrochemistry and soil pollution in a catchment affected by an abandoned lead-zinc mine: Functioning of a diffuse pollution source. Environ. Earth Sci. 2012, 4, 1179-1189. [CrossRef]

34. Jeon, S.-K.; Kwon, M.J.; Yang, J.-S.; Lee, S. Identifying the source of Zn in soils around a Zn smelter using pb isotope ratios and mineralogical analysis. Sci. Total Environ. 2017, 601, 66-72. [CrossRef] [PubMed]

35. Liuqiang, L.; Zhenhua, D.; Jinling, L.; Huina, L.; Hao, W. Distribution of heavy metals in surficial sediments from main mangrove wetlands of China and their influence factors. Acta Oceanol. Sin. 2008, 5, 159-164. [CrossRef]

36. Ji, Y.; Zhao, Z.; Wu, D. Distribution and bioavailability of seven heavy metals in mangrove wetland sediments in Dongzhai Harbor, Hainan Island, China. Chin. J. Appl. Ecol. 2016, 2, 593-600.

37. Wei, J.; Wang, Z.; Pan, L. Heavy metal contents and ecological risk assessment of surface sediments in Shankou mangrove wetland of Guangxi. J. Subtrop. Resour. Environ. 2019, 2, 28-33. [CrossRef]

38. Cai, M.; Wang, Y.; Qiu, C. Heavy metals in surface sediments from mangrove zone in Zhangjiang River estuary, South China. In Proceedings of the 2009 International Conference on Environmental Science and Information Application Technology, Wuhan, China, 4-5 July 2009; Volume 3, pp. 34-38. 
39. Chowdhury, R.; Favas, P.J.C.; Jonathan, M.P.; Venkatachalam, P.; Raja, P.; Sarkar, S.K. Bioremoval of trace metals from rhizosediment by mangrove plants in Indian Sundarban Wetland. Mar. Pollut. Bull. 2017, 124, 1078-1088. [CrossRef]

40. Kehrig, H.A.; Pinto, F.N.; Moreira, I.; Malm, O. Heavy metals and methylmercury in a tropical coastal estuary and a mangrove in Brazil. Org. Geochem. 2003, 34, 661-669. [CrossRef]

41. Harikumar, P.S.; Jisha, T.S. Distribution pattern of trace metal pollutants in the sediments of an urban wetland in the southwest coast of India. Int. J. Eng. Sci. Technol. 2010, 2, 840-850.

42. Baskar, B.; Biraja, K.S.; Jayappriyan, K.R.; Arockia, V.L.; Marimuthu, N.; Nandakumar, P.; Prabakaran, P. Assessment of heavy metal concentrations and associated resistant bacterial communities in bulk and rhizosphere soil of Avicennia marina of Pichavaram mangrove, India. Environ. Earth Sci. 2017, 1, 58. [CrossRef]

43. Mackey, A.P.; Hodgkinson, M.C. Concentrations and spatial distribution of trace metals in mangrove sediments from the Brisbane River. Aust. Environ. Pollut. 1995, 2, 181-186. [CrossRef]

(C) 2020 by the authors. Licensee MDPI, Basel, Switzerland. This article is an open access article distributed under the terms and conditions of the Creative Commons Attribution (CC BY) license (http://creativecommons.org/licenses/by/4.0/). 